\title{
THE BLOOD SUPPLY OF THE FEMORAL SHAFT
}

\author{
An Anatomical Study
}

\author{
P. Gowans Laing, Pembury, Kent
}

It is axiomatic that the surgeon must possess a detailed knowledge of the anatomy of the part on which he is to operate. In spite of the recent popularity of open reduction in the treatment of the fractured femoral shaft, little information is available on the details of its blood supply. Since the success of these operations depends, to a large extent, on a minimal interference with the blood supply of the bone the anatomy of the nutrient vessels is of considerable consequence. It was therefore decided to investigate the details of the blood supply of the femoral shaft.

\section{HISTORICAL REVIEW}

The first detailed account of the blood supply of the femur was given by Lexer, Kuliga and Turk (1904) in the course of a description of the supply of all the bones. These workers injected the arterial systems of newborn and adult post-mortem subjects with a mercuryturpentine emulsion and then took stereoscopic radiographs of the specimens. They also investigated the problem in a similar manner in amputation specimens. Lexer et al. found evidence of four main arterial systems supplying all long bones. These were namely: periosteal, diaphysial, metaphysial and epiphysial systems. The diaphysial or nutrient arteries supplying the femur were stated to be two in number and to enter the shaft of the bone in the region of the linea aspera. In children the superior vessel passed through the cortex in a downward direction and the inferior one in an upward direction. In adults both vessels are stated to pass through the cortex in an upward direction. These workers also state that the size of the arteries relative to the size of the bones decreases with increasing age.

The next detailed analysis of the problem was given by Anseroff (1934) as part of his researches into the blood supply of the long bones. This worker was only able to find evidence of three systems of nutrient arteries, namely, periosteal, diaphysial and epiphysial groups. With particular reference to the femur, Anseroff found that, although there were usually two nutrient arteries to the shaft, there was often only one. He also stated that the femora of children have more than two nutrient vessels in $\mathbf{2 3 \cdot 6}$ per cent. of cases while the same is true in only 8.7 per cent of adults. When considering this statement it is important to appreciate the way in which these figures were obtained. Anseroff determined the numbers and the topography of the nutrient vessels by the direct examination of the surfaces of macerated young and adult bones. The sources of error in this method will be discussed later. This worker agreed with Lexer that the nutrient arteries of the femur always enter the bone in the region of the linea aspera. He stated that in the usual arrangement one artery enters the upper third of the femur and one enters the lower part of the middle third.

\section{THE PRESENT INVESTIGATION}

Material and methods--The blood supply of the femur was examined in ten adult and in seventeen newborn subjects. In each case the femoral artery was injected with an aqueous suspension of barium sulphate. Care was taken to avoid the use of great pressure because it was found that the suspension would then fill both the arterial and the venous systems. The limbs were then dissected to determine the source of the nutrient vessels to the femoral shaft. The femora were then removed from the limbs and all soft tissue and periosteum was cleaned off them. The adult specimens were radiographed in antero-posterior and in lateral views. 


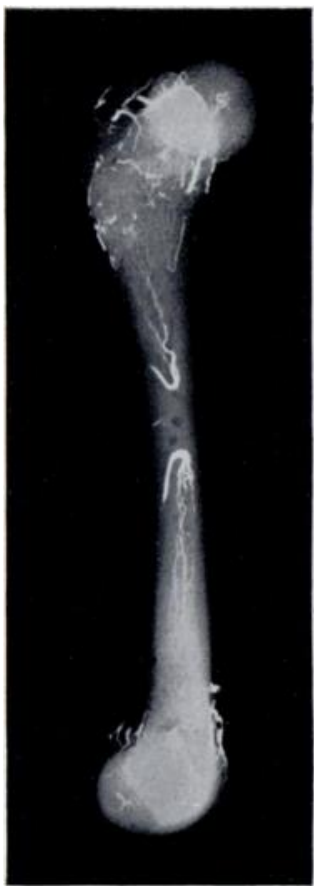

FIG. 1

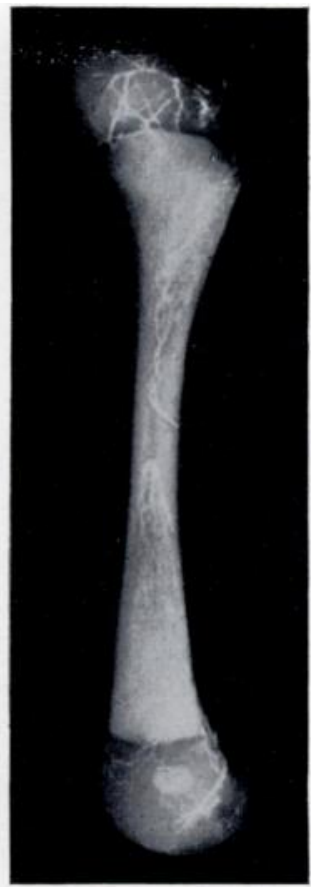

FIG. 2

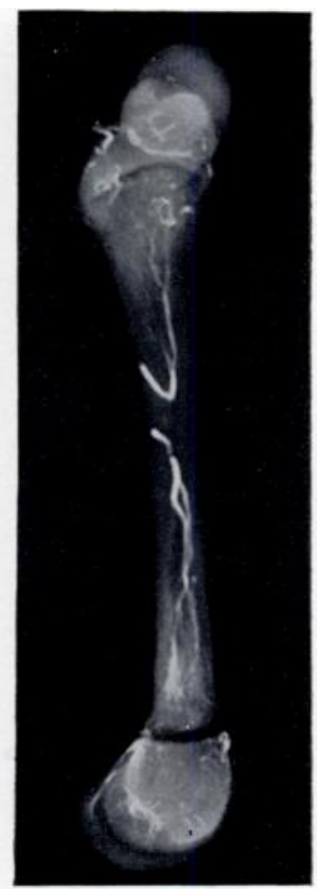

FIG. 3

Figure 1-Injected femur of a newborn infant. Two nutrient vessels are present. Note the venous foramina. Figure 2-Injected femur of a newborn infant. Both nutrient arteries ascend through the cortex. Figure 3-Injected femur of a newborn infant. Both nutrient arteries descend through the cortex.

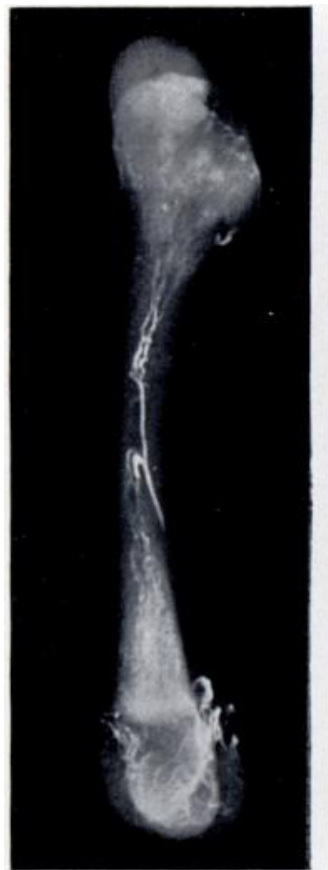

FIG. 4

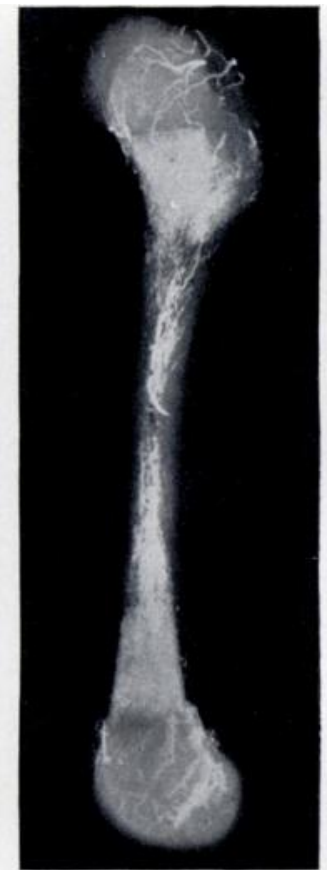

FIG. 5

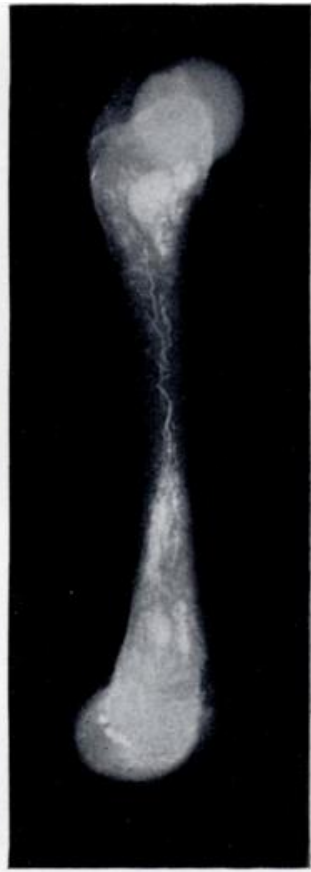

FIG. 6

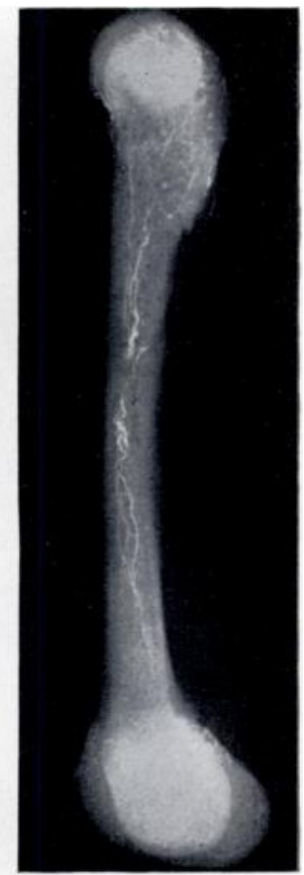

FIG. 7

Figure 4-Injected femur of a newborn infant. Only one nutrient artery is present. Note the long ascending intracortical course and the empty venous foramina. Figure 5-Injected femur of a newborn infant. Note the short ascending course of the single nutrient artery. Figure 6 -Injected femur of a newborn infant. Note the short descending course of the stem of the single nutrient artery. Figure 7-Injected femur of an eleven months old infant. The short nu trient stem is nearly horizontal.

vol. 35 B, No. 3, AUgust 1953 


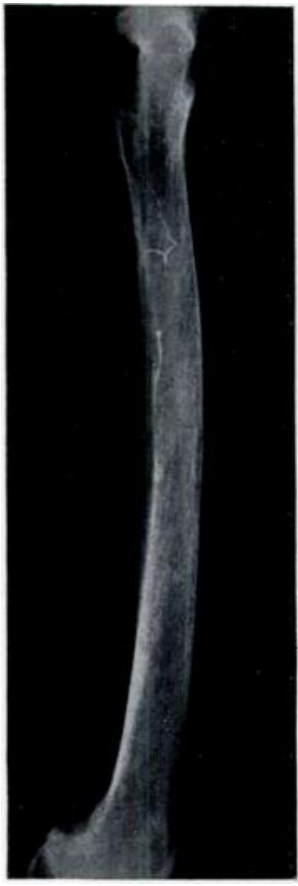

Fig. 8

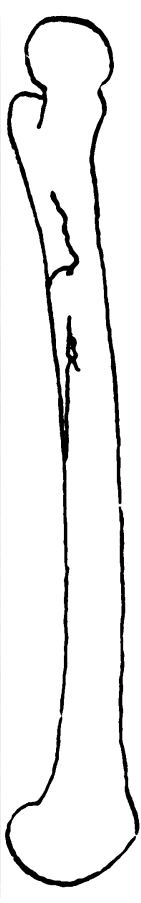

Figure 8-Injected adult femur showing two nutrient arteries, both entering the upper half of the shaft. Three out of ten adult femora examined were of this type. Figure 9-Injected adult femur showing two nutrient vessels. Note low position of the inferior nutrient. The large opacities were produced by rupture of small arteries. In only this one adult femur did a nutrient vessel enter the lower third of the shaft.

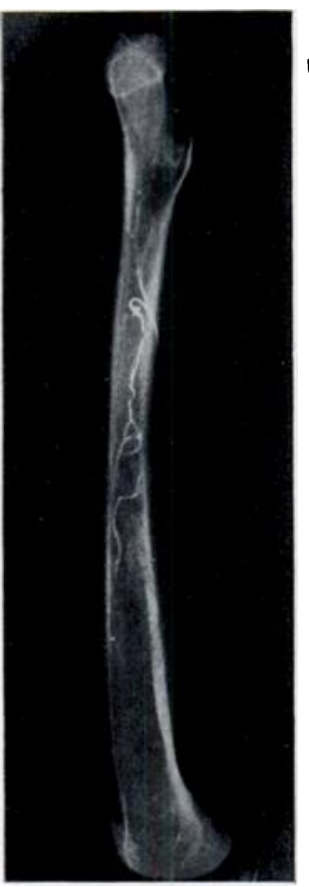

FIG. 10

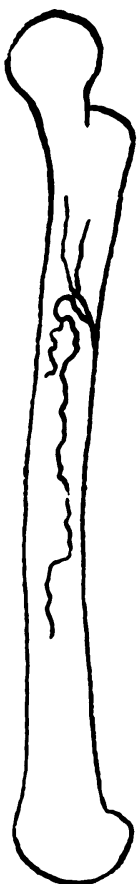

FIG. 11

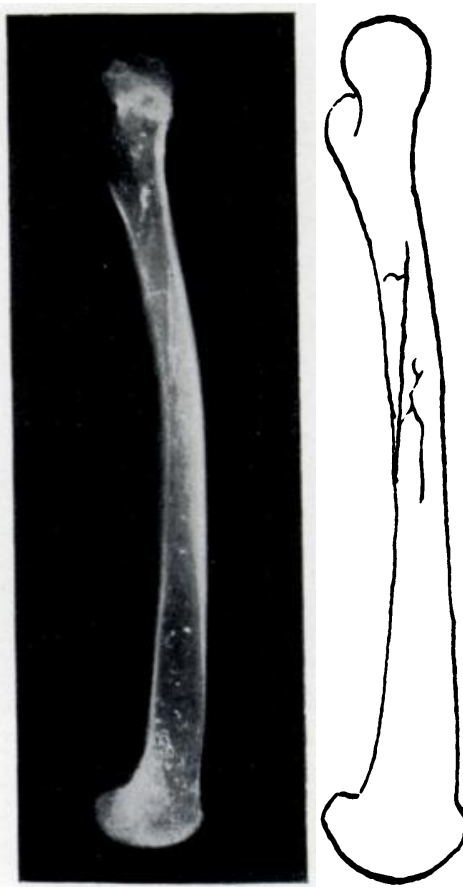

Figure 10-Injected adult femur showing one nutrient artery having a short transcortical course in the upper part of the bone. Five out of ten adult femora examined were of this type. Figure 11-Injected adult femur showing one nutrient artery having a long ascending passage through the cortex. The scattered opacities were produced by barium leaking through ruptured vessels. 
The bones from the stillborn and the newborn subjects were decalcified in 10 per cent nitric acid, neutralised in $\mathbf{1 0}$ per cent sodium carbonate and then radiographed. In the early stages of the investigation some of these smaller bones were not decalcified.

Findings-In all subjects the nutrient arteries arose as branches of one or other of the perforating branches of the arteria profunda femoris. In most cases the first perforating branch supplied one nutrient artery, usually the superior one, and the inferior nutrient arose from one of the remaining perforating vessels in an inconstant manner. In all cases the nutrient arteries entered the femoral shaft in the region of the linea aspera.

Seventeen femora from children under one year of age were examined. In eleven two nutrient arteries supplied the femoral shaft. The direction of these arteries through the cortex of the femur was found to vary. In eight cases the superior artery took a downward course until it reached the medullary cavity when it turned upwards, and the inferior one took an upward course through the cortex before turning downwards (Fig. 1). In two cases both arteries passed through the femoral cortex in an upward direction (Fig. 2), and in one case both took a downward course (Fig. 3).

In the remaining six children's femora only one nutrient artery supplied the shaft. In two of these the artery had a long ascending transcortical course (Fig. 4), and in one it had a short ascending course (Fig. 5). In two bones the artery had a short descending stem (Fig. 6), and in the last case, that of a child aged eleven months, the intracortical stem was short and horizontal (Fig. 7).

In the ten adult femora (Figs. 8 to 11 ) the nutrient arteries all passed through the femoral cortex in the region of the linea aspera and had an ascending course. The length of the transcortical course varied considerably from case to case.

In four bones two nutrient vessels entered the shaft and in three of these both arteries entered the upper half of the femur (Fig. 8). In one case (Fig. 9) the inferior artery was a small vessel and entered the lower third of the shaft while the superior one entered the middle of the shaft.

In six of the adult femora only one nutrient artery supplied the shaft. In five of these the vessel had a short transcortical course in the upper third of the bone (Fig. 10) and in one case (Fig. 11) the artery entered the cortex at the lower end of the middle third and had a long ascending passage through the cortex.

In all these cases the arteries branched as soon as they had reached the medullary cavity and these branches passed up and down the medullary cavity.

\section{DISGUSSION}

The findings presented here are not intended as a statistical analysis but are merely an indication of the variability of these nutrient arteries. It is obvious that a considerable number of femora have only one main nutrient vessel supplying the shaft. It is also notable that in most cases, even including the bones with two such vessels, no major artery entered the lower third of the shaft. In no case were more than two nutrient vessels found and it is possible that Anseroff mistook the quite large venous foramina of children for nutrient foramina. These can be seen in Figures 1 and 4 as quite separate entities, and are also seen in adults.

It thus emerges that the blood supply of the femoral shaft is in many ways similar to that of the tibial shaft which constantly has only one nutrient artery. The dangers of avascular necrosis in fractures of, and in operations on, the tibial shaft are too well known to bear repeating. From these experiences with the tibia we have learnt that, in the absence of the main nutrient artery, the epiphysial and periosteal arterial systems cannot always take over the nutrition of the lower fragment of the shaft and avascular necrosis or impaired osteogenesis results. A fracture through the junction of the upper and middle thirds of most of the femora investigated would have deprived the upper end of the lower fragment of its main blood

vol. 35 B, No. 3, AUgust 1953 
supply. It is of interest to calculate the effects of a fracture through the junction of the middle and lower thirds of the femoral shaft in cases having one nutrient artery with a long intracortical course (Fig. 12). As can be seen, this state of affairs would account for the cases of avascular necrosis of the lower end of the upper fragment occasionally met with in fractures of this type.

It is perhaps also worthy of note that the passage of a correctly fitting intramedullary nail in any one of the adult femora investigated would have destroyed most of the medullary branches of all the nutrient vessels in every case.
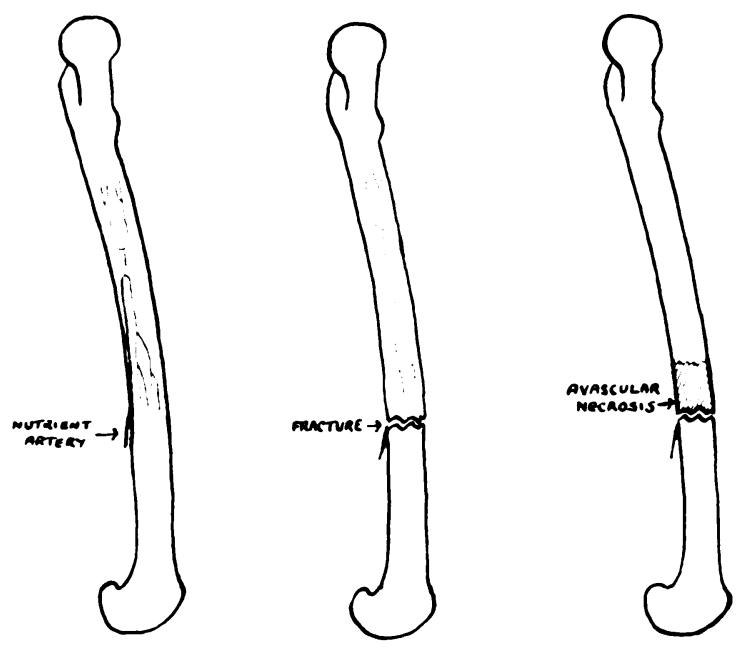

FIG. 12

Diagrammatic representation of the possible effect of a fracture on a femur with a single long nutrient artery.

The constancy with which the arteries enter the femoral shaft in the region of the linea aspera needs reiterating. It is obvious that the linea aspera should never be stripped of muscle attachments at operation. It is not quite so obvious that when plating the femur one should never put the plate on the anterior surface of the bone, for then the screws might well penetrate the linea aspera posteriorly and, if the femur in question has only one nutrient artery, could destroy the major source of blood to the shaft.

\section{SUMMARY}

1. Previous investigations into the blood supply of the femoral shaft are reviewed.

2. Details of the blood supply of the shaft of the femur in seventeen children under one year of age, and in ten adults, are given.

3. These findings are discussed with special reference to the fate of the femoral shaft after fracture or operation.

\section{REFERENCES}

Lexer, E., Kuliga, P., and TURK, W. (1904): Untersuchungen über Knochenarterien. Berlin: A. Hirschwald.

Anseroff, N. J. (1934): Die Arterien der langen Knochen des Menschen. Zeitschrift für Anatomie und Entwicklungsgeschichte, 103, 793. 